\title{
Under the radar: older women YouTubers and algorithmic influence ${ }^{1}$
}

\section{Melissa Bliss}

Queen Mary University of London

Contact_melissa.bliss@qmul.ac.uk

ORCID http://orcid.org/0000-0002-3637-3832

To cite this article: Melissa Bliss (2020): Under the radar: older women

YouTubers and algorithmic influence, Feminist Media Studies, DOI:

10.1080/14680777.2020.1783806

To link to article: https://doi.org/10.1080/14680777.2020.1783806

\section{Introduction}

Older women have been absent from considerations of YouTube's algorithmic structure on the visibility of videos made by people from marginalised groups. Older women are keen makers of YouTube videos in many genres but their contributions are less visible than those of younger women due to YouTube's algorithmic processes. These reflect and reproduce the power relations and inequalities of society (Dan McQuillan 2016) including the social devaluation of older women (Barbara Macdonald and Cynthia Rich 1985). This is in the wider context of online media research in which older women are still assumed to be reluctant content creators and motivated to share videos only with a family and close pre-existing friends (Susan M. Ferreira, Sergio Sayago and Josep Blat

${ }^{1}$ This is an Accepted Manuscript of an article published by Taylor \& Francis in Feminist Media Studies. Published online 22 June 2020. 
2017). My commentary here draws on my observations of 46 women YouTubers aged $60+$, their videos and their interactions.

One starting point for considering the influence of YouTube's algorithmic structures on older women's production practices and visibility is foregrounding the pervasive power of YouTube's algorithmically determined search and recommendation functions. It is well understood that Google, YouTube's owner, uses predictive analytics based on individual and collective characteristics and past watching habits to offer viewers a continual stream of videos they want to see, and at the same time deliver target audiences to advertisers. This is effective: $70 \%$ of watching time comes from these automated recommendations (Kevin Roose 2019).

This has material consequences for YouTubers and viewers as to visibility and produc- tion practices. YouTube appears to favour the most popular and gender normative content (Siqi Wu, Marian Andrei Rizoiu and Lexing Xie 2019; Sophie Bishop 2018). I noted older women's videos seem to less visible in the recommendations: they are unlikely to be recommended to viewers unless the video currently being watched is by an older person. Moreover, video production practices are shaped by beliefs about what will lead to higher rankings and larger audiences. The exact workings of the ever-evolving algorithm are commercially protected but information has been deduced from Google personnel (for example, Alex Beutel, Paul Covington, Sagar Jain, Can Xu, Jia Li, Vince Gatto, and Ed H. Chi 2018; Kevin Roose 2019) and spread through "algorithmic gossip" (Bishop 2019). These become part of what is considered legitimate or appropriate practices of making YouTube videos, whether or not the YouTuber explicitly intends them to improve their ranking. 
A different starting point for understanding algorithmic influence is to consider the benefits of being "under the radar." As Peggy Phelan argued, invisibility can be a protective space that insulates women from critical and controlling attention (Peggy Phelan 1993, 6). The older women YouTubers that I observed got a markedly less antagonistic response than that reported by younger women YouTubers. They received overwhelmingly positive comments and almost none that were critical of their ageing appearance, or their "inappropriate" behaviour, as they may have in other public spaces (Rachel Heinrichsmeier 2019). Even videos discussing enjoying sex attracted few hostile, ridiculing, or sexually explicit comments.

Older beauty vloggers, in particular, I noted, are likely to be part of loose interconnected networks of mutual support with other beauty vloggers. Beauty vlogging is a highly popular and, for some, lucrative genre. Beauty vloggers are commonly expected to be young women but there are many aged sixty and over. The networks I observed are less formal than Instagram pods (Caitlin Petre, Brooke Erin Duffy and Emily Hund 2019) and include more established and newer vloggers. They share mutual interests and reciprocally subscribe to each others' channels, comment on each others' videos, and name each other in their videos. One such case is Nathalie TheBeautyDiva, a more established older beauty vlogger with, at the time, over twenty-seven thousand subscribers, who posted a supportive comment to Claudeebeingclaudee, a newer vlogger with no subscribers: "I so enjoyed spending some time with you this morning with my first cup of coffee, I could spend all day with you. I loved this video, I hope you do more of these Lifesyle Favorite videos, I just love how you describe everything!!" (Claudeebeingclaudee 2016).

The ways in which the combining of predictive analytics and culture play out are complex (Dan McQuillan 2016). I have briefly delineated a few 
observations about the intersection of gender, age, and algorithmic-shaped culture. My aim is to ask a feminist media studies to include older women in our consideration of YouTube, and indeed other social networking sites. YouTube, despite its structures of inequality, is a place of older women's self-expression and mutual support.

\section{Disclosure statement}

No potential conflict of interest was reported by the author.

\section{Funding}

This work was supported by the Engineering and Physical Sciences Research Council (EPSRC) through the Media and Arts Technology Programme, a Research Councils UK Centre for Doctoral Training (EP/G03723X/1).

\section{References}

Beutel, Alex, Paul Covington, Sagar Jain, Can Xu, Jia Li, Vince Gatto, and Ed H. Chi. 2018. "Latent Cross: Making Use of Context in Recurrent Recommender Systems." In Proceedings of the Eleventh ACM International Conference on Web Search and Data Mining, 46-54. doi:10.1145/ 3159652.3159727.

Bishop, Sophie. 2018. "Anxiety, Panic and Self-Optimization: Inequalities and the YouTube Algorithm." Convergence 24 (1): 69-84.

doi:10.1177/1354856517736978. 
Bishop, Sophie. 2019. "Managing Visibility on YouTube through Algorithmic Gossip." NewMedia and Society 21 (11-12): 2589-2606.

doi:10.1177/1461444819854731.

Claudeebeingclaudee. 2016. "Lifestyle Favorites | January." January 15. http://www.youtube.com/ watch?v=U4WW55UcwRw

Ferreira, Susan M., Sergio Sayago, and Josep Blat. 2017. "Older People's Production and Appropriation of Digital Videos: An Ethnographic Study." Behaviour and Information Technology 36 (6): 557-574. doi:10.1080/0144929X.2016.1265150.

Heinrichsmeier, Rachel. 2019. '“So Long as There's Hair There Still': Displaying Lack of Interest as a Practice for Negotiating Social Norms of Appearance for Older Women." Ageing and Society 39 (7): 1360-1386. doi:10.1017/S0144686X17001544.

Macdonald, Barbara, and Cynthia Rich. 1985. Look Me in the Eye: Old Women, Aging and Ageism. London, UK: Women's Press.

McQuillan, Dan. 2016. "Algorithmic Paranoia and the Convivial Alternative." Big Data and Society 3 (2): 205395171667134. doi:10.1177/2053951716671340.

Petre, Caitlin, Brooke Erin Duffy, and Emily Hund. 2019. '“Gaming the System': Platform Paternalism and the Politics of Algorithmic Visibility." Social Media and Society 5 (4). doi:10.1177/2056305119879995.

Phelan, Peggy. 1993. Unmarked, the Politics of Performance. London, UK: Routledge. doi:10.4324/9780203359433.

Roose, Kevin. 2019. "YouTube's Product Chief on Online Radicalization and Algorithmic Rabbit Holes." The New York Times, 1-7. https://www.nytimes.com/2019/03/29/technology/youtube- online-extremism.html 
Wu, Siqi, Marian Andrei Rizoiu, and Lexing Xie. 2019. "Estimating Attention Flow in Online Video Networks." Proceedings of the ACM on Human-Computer Interaction 3 (CSCW). doi:10.1145/3359285. 